\title{
The Medical Director in Healthcare Institution: From Monitoring to Management for a Safer and Enhanced Quality of Clinical Care
}

\author{
Elkhoury Ziad and Kossaify Antoine*
}

Department of Cardiology, University Hospital Notre Dame des secours, Lebanon

*Corresponding author: Antoine Kossaify, Department of Cardiology, University Hospital Notre Dame des Aide, USEK, St charbel street, P.O.Box 3, byblos, Lebanon, Tel: +9613232192;

Email: antoinekossaify@yahoo.com

Mini Review

Volume 3 Issue 5

Received Date: August 24, 2020

Published Date: September 07, 2020

DOI: $10.23880 /$ jqhe- 16000180

\section{Abstract}

In a healthcare facility, the medical director is expected to monitor and manage the overall medical facets that may affect the institutional healthcare system; in this regard, the role of the medical director in regulating and improving the institutional healthcare system is predominant and should be a priority and a continuous concern of note, the role of the medical director is changing over time and it is evolving from what was a strictly "medical" function to an expanded set of roles which are more administrative and managerial rather than purely medical. The medical director position and function in a healthcare facility should inspire and enhance the spirit of professionalism, excellence and fairness, also the medical director is expected to ensure appropriate quality of care and patient safety and satisfaction, along with adequate filling and completion of medical records, medical teaching and training. In this document, we sought to make a quick review to highlight the impact of the Med on the healthcare system with an insight into management and improvement strategies.

Keywords: Medical Director; Healthcare; Institution; Quality of Care; Safety; Management

\section{Introduction}

Professionalism in medical facilities is a minimal requirement, and a high quality of care is essential, the issue at stake is simply the difference between life and death. The medical director (Med) has the general function of supervising the medical establishment rules, including the appropriate implementation of policies, protocols, procedures. Moreover, the Med classically establishes the link between physicians and the executive director (or general director) [1].

Though medical accountability is directly related to the attending physician proactive, the impact of the surrounding context on individual medical practice is significant, including organization, availability of physicians, quality of equipment, interdisciplinary and teamwork work, quality of nursing care; these factors are mainly correlated to the Med function and policy. Of note, the term "Medical Director" is mostly used in Europe and many other parts of the world, whereas the term "Chief medical officer" is mainly used in North America, however, both appellations refer to the same position and function [2]. In this document, we sought to make a quick review to highlight the impact of the Med on the healthcare system with an insight into management and improvement strategies.

\section{Responsibilities and Monitoring Strategies}

The medical field tends to become a turbulent environment due to extreme regulatory constraints and unmeasurable competitiveness. The Med must ensure an adequate environment of the working forces, notably the 
Physicians, given that the Med is theoretically liable of every process that may interfere directly or indirectly with the quality of care. Of note, the function of the Med is changing over time and it is evolving from what was a strictly "medical" function to a more extensive managerial function [3].

Professionalism in healthcare facilities is a minimal requirement and the Med is accountable for medical supervision and regulation of the overall medical facets that may affect the institutional healthcare system. The Med has to supervise and implement institutional policies, protocols and procedures, while coordinating with the Executive Director for better outcome. Issues related to professionalism, fairness, quality of care, patient safety and satisfaction, medical teaching and training, completion of medical records, continuous medical education, ethical issues and teamwork are the cornerstone parameters in the perspective of institutional development and improvement strategies [4].

In most healthcare facilities, physicians have frequently variable degrees of training and experience given their different background. One of the fundamental functions of the Med is to ensure that physicians have acquired appropriate certification, sufficient knowledge and skills before being enrolled as attending physicians in the institution. The monitoring strategies include the personal effort along with the sub-committees allocated in this perspective, and the main objective is to focus on patient safety, ensure advanced quality of care, avoid preventable medical errors, also the Med has to ensure that physicians engage in scientific events, comply with continuous medical education requirement. Table 1 shows the different parameters associated with physician professional record $[5,6]$.

\begin{tabular}{|c|c|}
\hline Qualification/documents & Monitoring of variables/documents \\
\hline Certifications & CV, Diploma and relevant certificates \\
\hline Experience and expertise & Years of practice, caseload, skills in advanced techniques \\
\hline Regular updating & CME certificates, training update (hands-on) certificates \\
\hline Academic and scientific profile & $\begin{array}{l}\text { Shadowing and training of students and fellows Publications, research, active } \\
\text { participation in scientific meetings, membership in national and international medical } \\
\text { societies }\end{array}$ \\
\hline Clinical performance & $\begin{array}{l}\text { Clinical outcome, administrative monitoring, feedback from referring physicians, patient } \\
\text { satisfaction survey. }\end{array}$ \\
\hline Professionalism and behavior & $\begin{array}{c}\text { Ethics, availability, teamwork, collaboration Commitment to the ethical and professional } \\
\text { principles }\end{array}$ \\
\hline
\end{tabular}

Table 1: Main parameters associated with physician professional record.

CME, continuous medical education; $\mathrm{CV}$, curriculum vitae [5,6]. The Med (and the affiliated sub-committees) must be capable of gathering, processing all necessary data, including budget and reimbursement issues, to ensure best medical practice. In this regard, it is recommended to use objective qualitative and quantitative indices (metrics) to evaluate physician performance and clinical outcome.

\section{Managerial and Organizational Tasks}

The Med must develop cooperation with other directions (nursing and staff direction, financial direction, medical committee, etc.), also he/she has to ensure cooperation among the different medical departments, physicians, paramedics and other working personnel, for a better teamwork and enhanced clinical outcome, with an institutional culture based on the principle of "patient first". The main issues to manage in this regard relate to patient safety and clinical outcome, quality of care, hospitalization rate and duration of stay, auditing and accreditation, standardization and research, ethical values.

\section{Patient Safety and Clinical Outcome}

The Med main role is to ensure patient safety and best appropriate clinical outcome, and he/she has to implement sufficient authority in this regard, and work on preventable medical errors, otherwise he may be transformed to a bystander Med. Performed by qualified appointees, regular audits on patient records and morbidity/mortality committees allow detecting and addressing any issue related to patient safety or any potential deficiency regarding quality of care. Medical record is the responsibility of the attending physician, the audit is based mainly - but not only - on the documentation found in the medical record [7].

\section{Quality Assurance and Improvement}

Quality assurance and quality improvement are optimally planned by a peer committee, and the Med is expected to 
approve and ensure implementation of quality improvement project within a timetable in order to evaluate results and to apply corrective measures. The Med has to monitor and manage the roadmap of any strategic planning, from idea to action, aiming to identify and address any potential factor of deficiency in any medical unit or division while targeting a patient-centered project [8].

\section{Hospitalization Volume and Duration of Stay}

Hospitalization rate and occupation percentage are essential parameters to estimate and adjust the flow of patients in the healthcare facility, also these factors are important for staffing and shift administration and scheduling. Moreover, hospitalization rate per physician is an important index to monitor, knowing that excessively low or high rate per operator may affect quality. Of note, some physicians may have a trend to unnecessarily prolong the hospital stay, and this fact is deleterious regarding beds availability, patients' circulation and the institution image [1].

\section{Internal Auditing and Accreditation}

Auditing, whether internal (i.e. peer committee, auditing committee) or external (i.e. accreditation organizations), allows identifying potential deficiencies in medical units and divisions. Besides, auditing putting a framework as the basis of a quality improvement program, in order to enhance all components of the clinical care process, with a persistent focus on a patient-centered plan. Passive auditing is performed via the registry system, however, active internal auditing or accreditation committees allow to detect weakness and to set up a timetable plan for improvement in a form of strategic planning, under the supervision of the Med [9].

\section{Standardization, Teaching, Research}

The need for adhere to guidelines regarding medical practice is the gold standard approach for better clinical outcome. Continuous medical education along with creation and implementation of protocols conform to guidelines allows enhancing quality of care and preservation of resources. Supervising and training of juniors is also a task of senior physicians, in a framework of team-based collaboration, in order to well prepare the next generation with supervision and systematic quality checking. The Med has a fundamental role of enhancing and implementing such a culture and practice in the healthcare facility. Engaging in medical research is another track that must be encouraged, especially in academic medical facilities, and the Med has the role the facilitate re-use of medical data within ethical protocols and consent agreements for this purpose $[1,10] \ldots . . .$.

\section{Relational and Ethical Issues}

The Med may play a crucial role for teamwork enhancement, with a unifier profile based on equity, ethics, and professionalism. The Med has to control and restrict any potential abusive behavior of physician(s) in the healthcare facility. The Med has to apply sufficient authority when required to suppress ethical breach, otherwise, his function is simply relinquished and he may be transformed to a bystander M.Ed. Of note, many of the tasks of the Med could be deputized to the medical and units managers on a case by case decision. Figure 1 shows managerial and organizational factors on which the Med has to work on $[1,11,12]$.

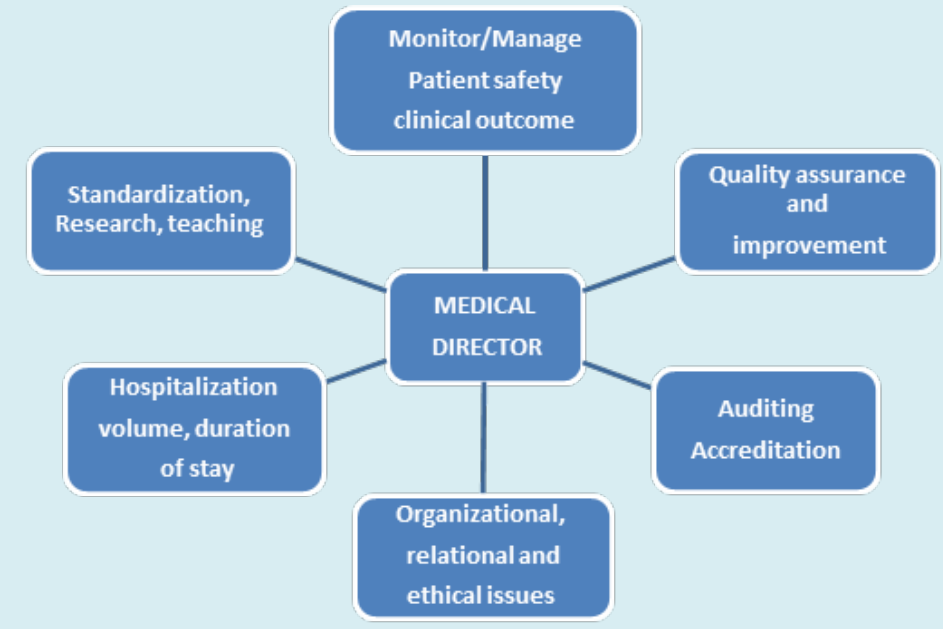

Figure 1: Role of the medical director and related managerial and organizational factors. 


\section{Challenges and Commitment}

In an environment made turbulent by financial interests and aggressive competitiveness, the MeD must promote respect and ethical values based on fairness and integrity, promoting the spirit professionalism in the institution. The Med has the commitment to promote an institutional culture based on the principle of "patient first". In this regard, an appropriate relationship between the Med and the attending physicians is essential and must be based on trust, respect and professionalism rather than excessive authority or unnecessary conviviality. In addition, the Med has to encourage multidisciplinary work - when necessary- for a better quality of care [13].

The Med must have an "evolutionary" or "revolutionary" mind to deal with every emergent situation, whether sanitary or financial or other (i.e. Covid-19, national political and/or economical instabilities, etc.), especially when the issue at stake is the institution sustainability (do-or-die). Accordingly, the Med has to predict and quickly define any threat in this regard, to measure the short and/or long term consequences, analyze the whole context, apply available tools for adjustment, also he/she has to hold brain-storming meetings to address any issue and avoid or limit potential damage. The Med must have a three-dimensional mind, thinking globally and applying locally in order to apply feasible management strategies even though some conditions (financial, technical, etc.) are not sufficiently or timely available in a respectable but less than perfect medical domain $[13,14]$.

The Med must have and/or develop the sense of communication, for negotiation with different stakeholders' representatives (business, insurances and other third party payers, etc.). In addition, innovation and acquirement of new technologies and techniques must be well analyzed for its cost-effectiveness, while targeting quality of care and the prestigious image of an advanced healthcare facility. According, the Med has to be involved in the discussion of the institutional budget and financial capabilities [14].

The Med is supposed to update administrative regulations, also he/she must ensure obtainability of adequate and updated equipment, and allocation of highquality paramedical assistance (nursing, secretaries, etc.), aiming to ensure professional standards in performance. The Med is expected to develop and implement a strategic planning, with the participation of the different medical divisions and units, guided by a checklist and metrics of quality. Innovation in healthcare facility is a cornerstone for institutional sustainability and development, in order to keep updated with latest technology and techniques, also digital technology brings significant challenges that employees and physicians must keep up with it $[1,15]$.

\section{Conclusion}

The Med represents a local health governor, ensuring supervision and improvement of the institutional healthcare system. The Med must be able to gather essential and accurate information about each medical unit and division in order to better organize and prioritize issues, such as patient safety and clinical outcome. The Med has to promote high-quality of medical care, with endorsement of education, training and research in a rapidly evolving domain. The Med must enhance and implement strategic planning for the institution, ensure appropriate equipment and standardization of medical performance. Moreover, the Med must have communication skills that facilitate effective collaboration among physicians, with a spirit of teamwork, ethics and equity, and with a responsible profile to implement a patient-centered quality of care that is safe, efficient and equitable.

\section{References}

1. Kossaify A, Rasputin B, Lahoud JC (2013) The function of a medical director in healthcare institutions: a master or a servant. Health Serv Insights 6: 105-110.

2. O'Leary KJ, Afsar Manesh N, Budnitz T, Dunn AS, Myers JS (2011) Hospital quality and patient safety competencies: development, description, and recommendations for use. J Hosp Med 6(9): 530-566.

3. Maddux FW, Maddux DW, Hakim RM (2008) The role of the medical director: changing with the times. Semin Dial 21(1): 54-57.

4. Birrer RB (2002) The physician leader in health care. What qualities does a doctor need to be an effective organizational leader?. Health Prog 83(6): 27-30.

5. Norcini JJ (2003) Peer assessment of competence. Med Educ 37(6): 539-543.

6. Fried GM, Feldman LS (2008) Objective Assessment of Technical Performance. World J Surg 32(2): 156-160.

7. Manser T (2009) Teamwork and patientsafety in dynamic domains of healthcare: a review of the literature. Acta Anaesthesiol Scand 53(2): 143-151.

8. Taylor BB, Parekh V, Estrada CA, Schleyer A, Sharpe B (2014) Documenting quality improvement and patient safety efforts: the quality portfolio. A statement from the academic hospitalist taskforce. J Gen Intern Med 29(1): 214-218.

9. Burn S, D Cruz L (2012) Clinical audit-process and outcome for improved clinical practice. Dent Update 39(10): 710-714. 
10. Vastola P, Tosolini G (2005) Quality improvement, training and clinical governance: the role of the chief medical officer and his staff. Ig Sanita Pubbl 61(2): 213233.

11. Rathert C, Fleming DA (2008) Hospital ethical climate and teamwork in acute care: the moderating role of leaders. Health Care Manage Rev 33(4): 323-331.

12. Kossaify A, Hleihel W, Lahoud JC (2017) Team-based efforts to improve quality of care, the fundamental role of ethics, and the responsibility of health managers: monitoring and management strategies to enhance teamwork. Public Health 153: 91-98.

13. Layman EJ, Bamberg R (2006) Coping with a turbulent health care environment: an integrative literature review. J Allied Health 35(1): 50-60.

14. Gabel S (2011) Power in the role of the medical director: what it is and how to get more. Adm Policy Ment Health 38(3): 211-214.

15. Schnelle JF (1995) Total quality management and the medical director. Clin Geriatr Med 11(3): 433-448. 